\title{
CONF-900466--86
}

\section{IN-SITU PORE STRUCTURE ANALYSIS DURING AGING AND DRYING OF GELS}

Douglas M. Smith", Pamela J. Davis*, C. Jeffrey Brinker"*

"UNM/NSF CENTER FOR MICRO-ENGINEERED CERAMICS, University of New

Mexico, Albuquerque, NM, 87131, "Division 1846, Sandia National

Labsratories, Albuquerque, NM, 87185

ABSTRACT

SAND- $-90-2203 \mathrm{C}$

DE91 000044

The use of NMR relaxation measurements for the in-situ study of pore structure evolution during gel aging and drying is illustrated. The change in the pore size distribution and surface area of both wet and dried gels is examined as a function of aging conditions including temporal aging, thermal aging, changing $\mathrm{pH}$, and changing pore fluid. The effect of pore fluid $\mathrm{pH}$ on dissolution/reprecipitation in ordered packings of monodisperse silica spheres is also examined as a model system for particulate gels. As expected, the pore size distribution narrows with increasing time of treatment in high $\mathrm{pH}$ pore fluids. Interpretation of high $\mathrm{pH}$ results for the wet state is complicated by a microporous layer which forms on colloidal silica resulting in significantly larger wet surface area as compared to the final dried material. Narrowing of the pore size distribution, which is of interest for maximizing drying rates, is maximized in the least time by using either high $\mathrm{pH}$ or repeated ethanol washes for the base-catalyzed gel (B2) used.

\section{INTRODUCTION}

A major feature of sol-gel processing is the large degree of variation in pore siructure (i.e., mean pore size, pore size distribution, surface area, porosity, etc.) that occurs both during processing and in the final product (coating, fiber, aerogel, or xerogel). Pore structure control at all processing stages is of great practical interest since pore structure plays an important role in fixing the highest drying rate that can be achieved without cracking (xerogels), sintering temperatures (dense glasses), and physical properties such as the refractive index or adsorption capacity. By varying process parameters such as temperature, pore solvent, $\mathrm{pH}$, aging time, etc., large changes in the final structure may be realized. However, the relationship between these parameters and pore structure is unclear and may be only elucidated with in-situ characterization techniques. 
The ability to monitor pore structure changes during aging and drying is complicated by the nature of sol-gel processing. In general, changes during processing have been inferred from the final dried gel since for traditional pore structure analysis (nitrogen adsorption/condensation and mercury porosimetry), the sample must be dried before analysis. However, chemistry and structure continue to evolve during aging and drying and the interpretation of how a parameter affects the final pore structure is not straightforward. The few studies of pore structure evolution in gels during processing use either scattering (SAXS, SANS), thermoporometry, NMR relaxation, or magnetic resonance imaging (MRI).

Scattering has primarily provided information on nucleation and growth mechanisms before the gel point $[1,2]$ and/or the structure of the final xerogel or aerogel. The use of scattering for in-situ pore structure analysis suffers from limited length scales (1-20 nm, SAXS only), contrast problems, relation of results to pore size, multiple scattering, and errors resulting from desmearing. However, the approach is quick, allows extraction of all length scales at once, and accesses closed porosity.

Thermoporometry provides pore size information from comparison of melting and solidification thermograms (i.e., the freezing/melting temperature of pore fluid is a function of pore size) $[3,4]$. This approach is useful for determining pore size distribution with pores in the size range of 1.5 to $150 \mathrm{~nm}$ but suffers from limitations regarding its use for sol-gel processing. These include the fact that the pore fluid must be very pure (requires multiple washing which changes gel structure], is nonisothermal, and the volume changes associated with phase change could significantly affect the structure of the fragile gels. In addition, the nature of the thermoporometry experiment precludes the continuous study of changes in a single sample as it undergoes various aging and drying steps.

NMR spin-lattice relaxation measurements have proven to be a valuable tool for analyzing the pore structure of wet solids $[5,6]$. This technique is well suited for in-situ studies of gel structure as it is nonintrusive in the sense that the pore fluid is used as the probe, high purity fluids are not required, and the temperature is held constant. Pore size and surface area information are obtained from the fact that fluid near a surface will undergo spin-lattice (T1) and spin-spin relaxation (T2) at a faster rate than for the bulk fluid. From the two-fraction, fast exchange model, the measured T1 or T2 is reiated to the pore size by [7]:

$$
\frac{1}{T_{1-2}}=\frac{1}{T_{1-2 b u l k}}+\frac{\beta}{r_{\text {pore }}}
$$

From relaxation measurements of fluid in the pores and the bulk fluid, the pore size (which is actually the hydraulic radius or twice the pore volume to surface area ratio) maybe obtained if the surface interaction parameter, $\beta$, is known. For a porous solid, a distribution of relaxation times exists which must be extracted from the relaxation data. The principle advantage 
of NMR relaxation measurements is the completely non-intrusive nature of the measurements, the short times required for analysis, and the ability to study sol-gel processing from the initial sol precipitation to the final dried gel.

The pore structure techniques described above employ the implicit assumption that the sample is homogeneous spatially. This may or may not be true for the initial gel and final dried gel and is almost certainly not true during processing. However, the ability to obtain the spatial distribution of pore structure would be of great utility for understanding the basic physics of processing as well as designing materials with tailored spatial distributions for applications such as optics and catalyst supports. Recently, Ewing and co-workers [8] have combined magnetic resonance imaging (MRI) and NM'R relaxation to obtain the spatial distribution of porosity with spatial resolution of $60 \mu \mathrm{m}$ and the spatiallyaveraged pore size distribution in a silica gel during drying. However, MRI is relatively slow which may limit its utility for sol-gel processing (i.e., many processing steps occur on shorter timescales than the one hour required for a single image). To overcome this problem, Majors, et al. [9] have demonstrated a scheme for radially symmetric samples which reduces imaging time to less than one minute. The exchange of D2O and $\mathrm{H} 2 \mathrm{O}$ in a zirconia pellet was studiad and radial proton concentration distributions ( $<1$ minute per image) were extracted which compared well to diffusion theory. By using relaxation-weighted imaging, the actual spatial distribution of the pore size distribution could be obtained.

\section{BACKGROUND-PROCESSING EFFECTS ON GEL PORE STRUCTURE}

A number of treatments may be used to reduce drying time and tailor final pore structure. A common approach is to place the gel in a different solution before drying [10]. High pH solutions have been used to enhance dissolution/reprecipitation to promote neck formation between particles and thus strengthen the gel matrix. This will reduce shrinkage during drying and result in a larger final pore size. Alternatively, pore fluid may be exchanged with another solvent. Mizurio, Nagata and Manabe [11], interested in finding ways to reduce drying times needed to obtain monoliths, placed silica gels in various solutions including neutral water. The objective was to strengthen the matrix by replacing $-\mathrm{OR}$ with $-\mathrm{OH}$ and increase Si-O-Si bonds. These are only a few examples of structure modification and a more complete revievs is presented elsewhere [12].

In the studies described above, the effect of the various processing parameters on pore structure was assumed (i.e., no direct measurement of pore structure was attempted). Quinson, Dumas, and Serughetti [13] applied thermoporometry to obtain pore structure information for a single acid-catalyzed silica gel. Special care was taken to ensure that the 
sample was repeatedly washed such that it only contained water and a narrow pore size distribution with radius of $2.5 \mathrm{~nm}$ was observed. Quinson and co-workers [15] employed thermoporometry to ascertain the effect of various pore fluids on the wet gel structure of a titania and a zirconia gel.' Because thermoporometry was employed, samples were all analyzed with the same final pore fluid (decane). The pore volume and pore size were shown to increase with aging and solvent polarisability. Glaves and coworkers [15] have used NMR relaxation measurements to monitor pore structure evolution of iwo base-catalyzed silica gel samples aged in either ethanol or eihanol/KOH solutions. In addition to the variation of the wet gel structure with pore fluid, the changes in both surface area and pore size distribution during drying were obtained. As expected for the base-aged sample, the final pore volume was higher and the final surface area was lower than the sample aged in ethanol. However, the surface area and pore size distribution variations during drying suggest more complex physics and chemistry than is normally assumed.

\section{EXPERIMENTAL}

Silica gels were prepared via a two-step base-catalyzed procedure (designated B2) as described by Brinker et al. [16]. In the first step tetraethyl orthosilicate (TEOS), ethanol, water and $\mathrm{HCl}$ (molar ratios $1: 3: 1: 0.0007)$ were heated under constant reflux for 1.5 hours. In the second step, $1 \mathrm{ml}$ of $0.05 \mathrm{M} \mathrm{NH} 4 \mathrm{OH}$ was added to $10 \mathrm{ml}$ of the TEOS stock solution. The mixture was then allowed to gel (2 hours) in $5 \mathrm{~mm}$ NMR tubes and age in the mother liquor for 22 hours. In addition to gels, ordered packings of monodisperse sphores were prepared. $130 \mathrm{~nm}$ silica spheres were synthesized following Stober et al., [17] and ordering was conducted via centrifugation. Aging was done in $\mathrm{KOH}$ solutions for $24 \mathrm{hrs}$.

To study the effect of pore fluid on aging, samples were prepared and aged in mother liquor, ethanol, water, or consecutive ethanol-water baths. After the initial 22 hour aging period in mother liquor, gels were forced out of the NMR tubes and placed in the aging solvent. Aging was conducted at either ambient, $30 \mathrm{C}$, or $60 \mathrm{C}$. After aging, samples were placed in NMR tubes and allowed to equilibrate at $30 \mathrm{C}$. NMR measurements on pore fluid were performed at $20 \mathrm{MHz}$ on a Spin-lock CPS-2 pulse NMR using a $180^{\circ}-\tau$ 9,0 pulse sequence with $30 \tau$ values varied non-uniformly from $100 \mu \mathrm{s}$ to $Q$ s. The T1 distribution was determined with regularization [18]. To relate T1 to pore size, the "two-fraction fast-exchange" model [7] was used and the value of the surface-interaction parameter, $\beta$, was found via relaxation experiments on partially saturated samples [19]. Nitrogen sorption was used to obtain surface area [5-point BET analysis $\left(0.05<P / P_{0}<0.31, N_{2}\right.$ area $\left.\left.=0.162 \mathrm{~nm}^{2}\right)\right]$ and volume [1 point, $\left.P / P_{O}=0.995\right]$ of dried gels.

Micrographs were obtained using a Hitachi S-800 SEM. 


\section{RESULTS AND DISCUSSION}

The most common aging scheme is to allow the sample to remain in its mother liquor for an extended time after the gel point. During this time, chemical and structural changes continue which have been categorized as [10]: (1) hydrolysis or the reverse, reesterification, (2) polymerization, or depolymerization, (3) syneresis, and (4) coarsening (dissolution/reprecipitation). The effect of aging time on the wet pore size distribution (PSD) of B2 silica gel is illustrated in Figure 1. PSL's are presented as $\mathrm{dV} /$ dlogr plots so that the integrated area under the plot directly corresponds to the pore volume. Since these samples did not exhibit microporosity, it is apparent from Fig. 1 that the total pore volume is approximately constant during aging. This result is quite different from that reported for titania and zirconia gels [14] which indicated a large increase in pore volume with aging. With increased aging, one would expect a higher degree of condensation (i.e. an increase in Q4 silicon) and increased dissolution/reprecipitation which could lead to greater mechanical strength, and thus allow greater drying rates without cracking. However, even though the mean pore size is relatively constant, the narrower pore size distribution with increased aging time should also allow faster drying rates since stress during drying will be reduced [12].

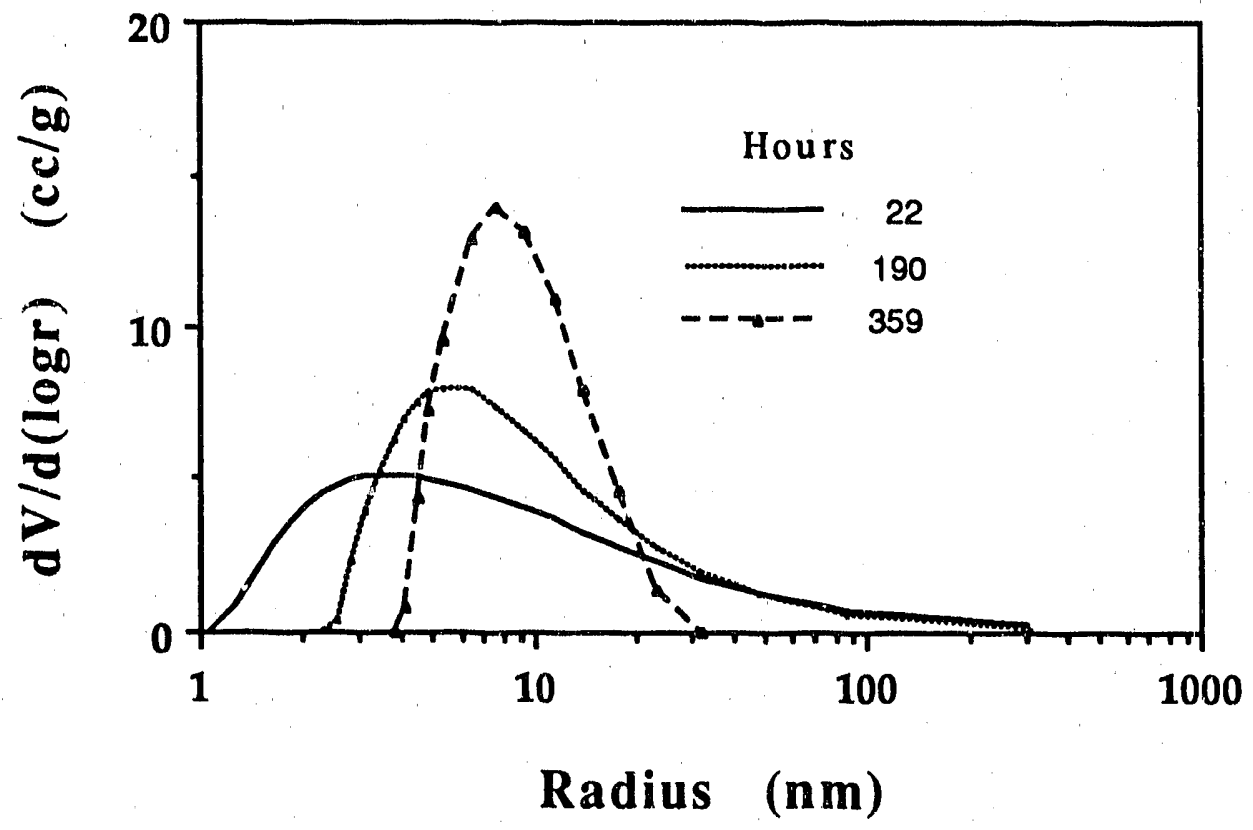

Figure 1 Effect of aging time in mother liquor at $30 \mathrm{C}$ on the wet gel pore size distribution.

Since the time required to narrow the PSD is large, investigators often age gels at elevated temperature. This effect is illustrated in Figure 2 for gels aged at either 30 or $60 \mathrm{C}$. At the higher temperature, the mean pore size increases by a factor of two over that of the $30 \mathrm{C}$ sample despite an aging time which is almost two orders of magnitude shorter. 


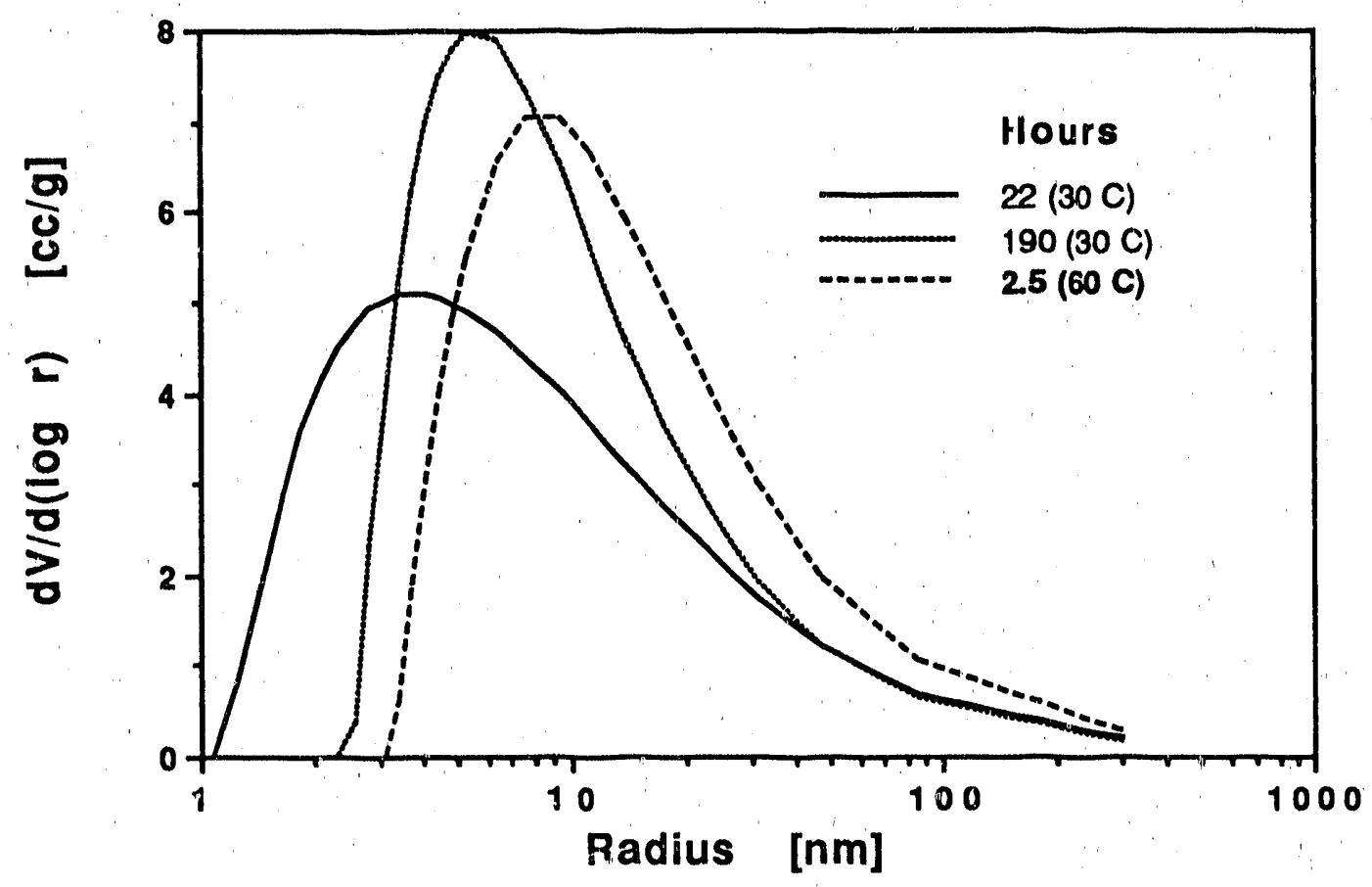

Figure 2 Effect of aging temperature on wet gel pore structure.

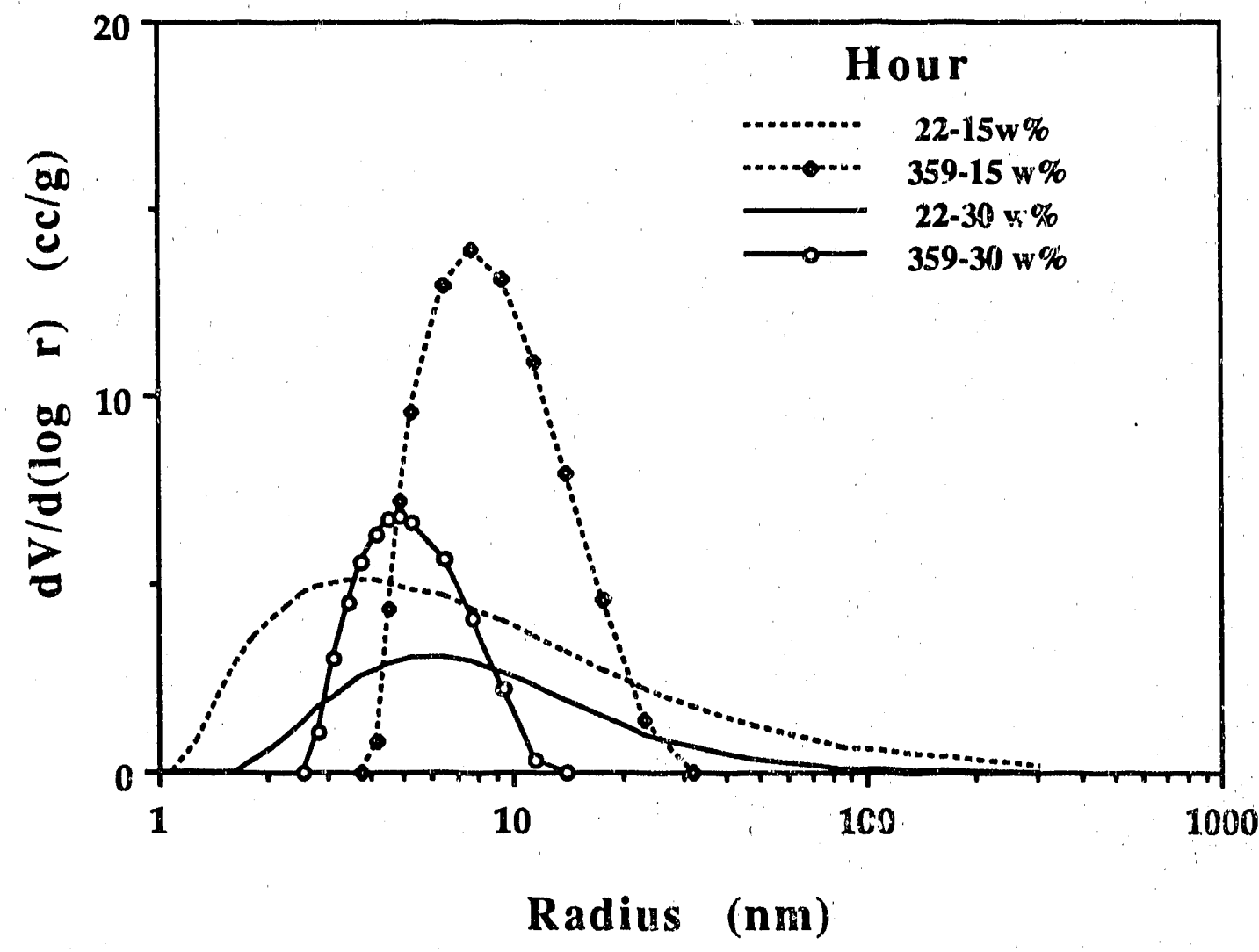

Figure 3 Effect of wet gel solids content on wet gel pore structure.

The effect of solids content on the wet gel PSD during temporal aging is illustrated in Figure 3. In general, the narrowing of the PSD with time is independent of solids content as both $15 \%$ and $30 \%$ exhibit similar broad distributions after 22 hours at $30 \mathrm{C}$ which then narrow after extended 
time. As expected, the area under the PSD (i.e., total pore volume) decreases with increasing solids content and the long time behavior appears to favor a smaller mean pore size with increasing solids content.

When a gel is repeatedly washed with ethanol, a dramatic narrowing of the pore size is observed. This narrowing is illustrated in Figure 4 and shows a much narrower PSD after 5 washings (120 hours total) than the gel simply aged in mother liquor for a comparable time (see Fig.1). The reasons for this result are unclear since one would expect decreased condensation and coarsening rates with ethanol as compared to mother liquor or water. This ethanol washing effect could be related to the increased concentration of Si-D.R groups at the pore surface.

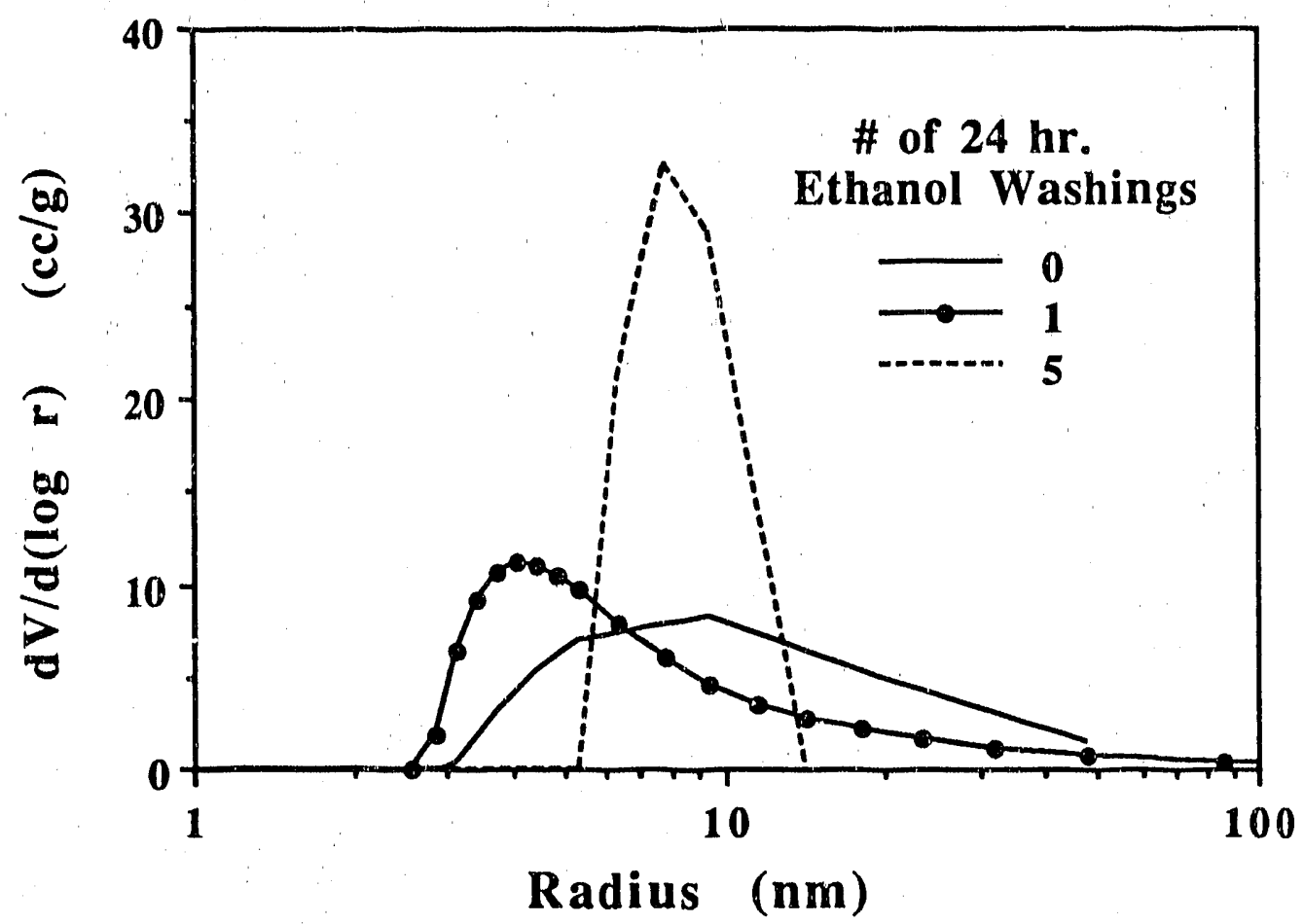

Figure 4 Effect of repeated ethanol washings on wet gel pore structure.

High $\mathrm{pH}$ solutions are often employed to promote dissolutionreprecipitation (Ostwald ripening) in particulate gels to increase pore size and matrix strength. To quantitatively illustrate this effect, ordered packings of monodisperse spheres were prepared since these packings have a known geometry and coordination number. The ordered nature and uniform particle size is apparent in Figure 5. The packings were infiltrated with $\mathrm{KOH}$ solutions of various strengths and the surface area was measured in solution after 24 hours as a function of $\mathrm{pH}$ (see Figure 6). With increasing $\mathrm{pH}$, one would expect a decrease in surface area as both solubility and dissolution rate increase. However, a surface area increase is observed. This could be due to either microporosity in the toroidal regions between particles, if dissolution is occurring as large oligomers rather 
Figure 5 SEM micrograph of a sphere packing fracture surface before aging.

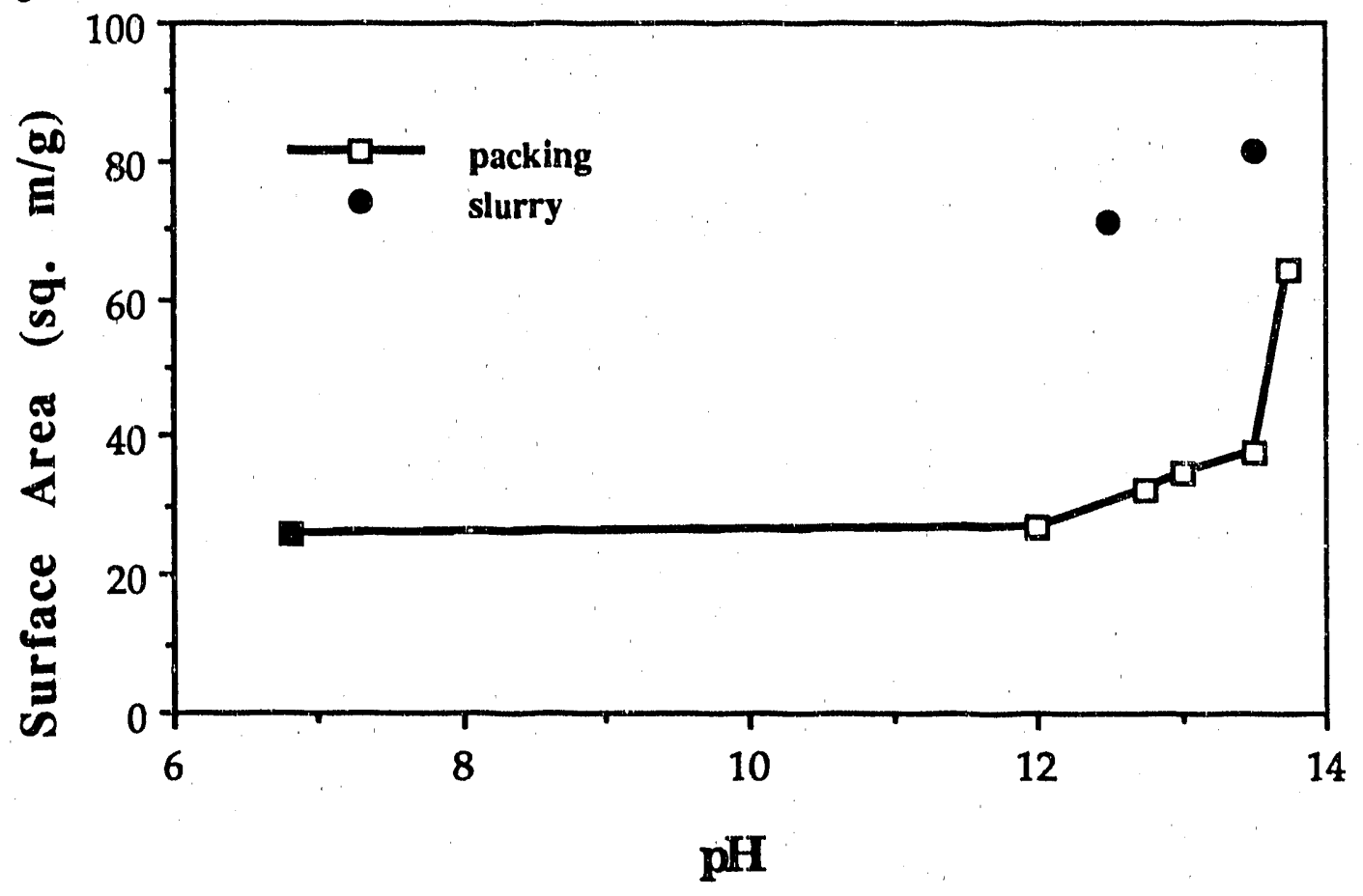

Figure 6 Effect of aging fluid $\mathrm{pH}$ on sphere packing and sphere slurry surface area.

than monomer, or as a result of a microporous gel layer occurring over the entire surface of the particles at high $\mathrm{pH}$. Upon drying at $110 \mathrm{C}$ under vacuum, the nitrogen surface area was found to be approximately $26 \mathrm{m2} / \mathrm{g}$ and independent of $\mathrm{pH}$.

To ascertain which mechanism caused this surface area increase, a similar experiment was performed on a slurry of spheres in order to prevent precipitation in regions of negative curvature. At a given $\mathrm{pH}$, an even larger surface area increase for the slurry is observed. To ensure 
that this surface area increase was only due to the particles and not related to dissolved silica, the spheres were separated from the solution via centrifugation and the experiment repeated on the solution. Negligible surface area was noted and therefore, we attribute the increase in surface area to the formation of a microporous gel layer on the surface of silica spheres. The reason that the increase is less for the packing is the result of decreased surface available for the gel layer in the region of the particle necks. Further evidence for this microporous gel layer on colloidal silica at high $\mathrm{pH}$ has been obtained from SAXS and charge density measurements [20]. We have not observed this effect for Cab-O-Sil fumed silica or for Stober spheres heated at $750 \mathrm{C}$.

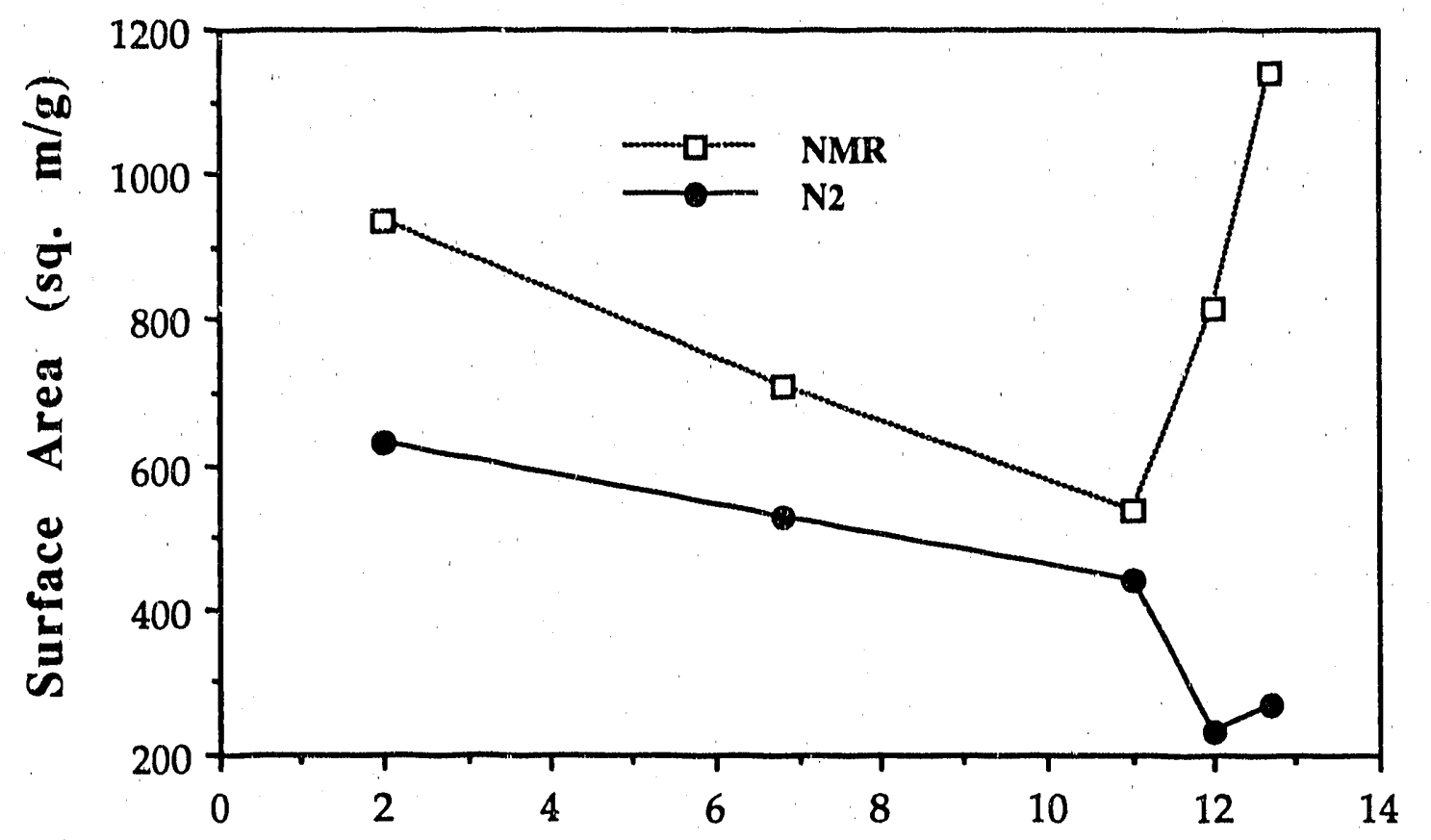

\section{pH}

Figure 7 Effect of $\mathrm{pH}$ on the wet and dry surface area for $\mathrm{B} 2$ gels.

In assessing the effect of pore fluid $\mathrm{pH}$ on the surface area of wet B2 gels (after 24 hours of aging), a surface area increase is observed for $\mathrm{pH}$ 12 and greater (see Fig. 7). We attribute to the same mechanism as for the colloidal sphere packing described previously. The higher surface area at $\mathrm{pH} 2$ as compared to neutral $\mathrm{pH}$ could be the result of the very low dissolution rate at this $\mathrm{pH}$ [21] which would serve to slow dissolution and repreciptation and thus lead to higher surface area. This result is supported somewhat by the nitrogen surface areas obtained after drying. At lower pH's, the dry surface areas parallel the wet values but at $\mathrm{pH} 12$ and greater, the collapse of this microporous gel during the final stages of drying leads to a large difference between wet and dry values. The low surface areas and high pore volumes (not shown) of these gels are also consistent with increased dissolution/reprecipitation at high $\mathrm{pH}$. A similar result was previously observed by Glaves and co-workers [15] for 
B2 gels aged in ethanol/KOH solutions. The change in PSD after 24 hours with these different solutions is illustrated in Figure 8. Simpiy placing the gel in water serves to narrow the PSD considerably. Increasing the $\mathrm{KOH}$ concentration increases the degree of PSD narrowing and at a $\mathrm{pH}$ of 12.7, the PSD is considerably narrower than that obtained with either temporal aging for 359 hours, thermal aging, or ethanol washing. Despite the large differences in the wet gel PSD, the pore size distribution of the final dried gel (which was subsequently filled with water for NMR pore size analysis) was relatively independent of pore fluid (see Fig. 9).

Between $\mathrm{pH}$ of 7 and 12.7, the mean pore size only varied from 2 to $2.5 \mathrm{~nm}$.

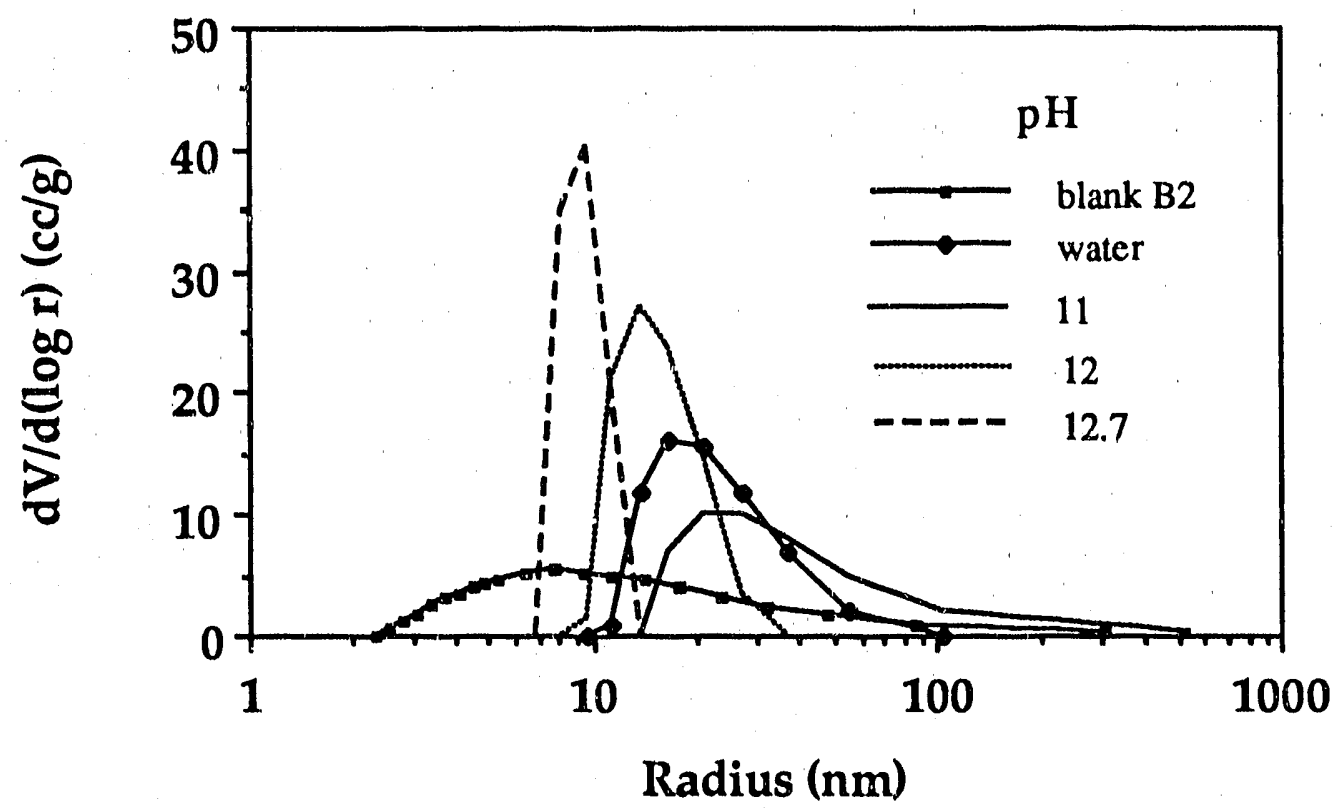

Figure \& Effect of aging B2 gels in high $\mathrm{pH}$ pore fluids on wet gel pore structure.

While studying the effect of ethanol washing, we noticed that the wet surface area increased when placed in ethanol and decreased when it is placed in water. Figure 10 illustrates the variation of surface area (both wet and dry) as a function of the final fluid as a gel is processed through a series of baths. This result was unexpected and raises the question concerning whether "surface" is being created by the ethanol (i.e., breaking Si-O-Si bonds) or whether Si-O-R groups on the pore surface result in a larger apparent surface area (both wet and dry). This question is still under investigation although preliminary silicon NMR and Raman results indicate that some Si-O-Si bonds are broken (i.e., the Q3/Q4 ratio varies depending upon the final pore fluid). 


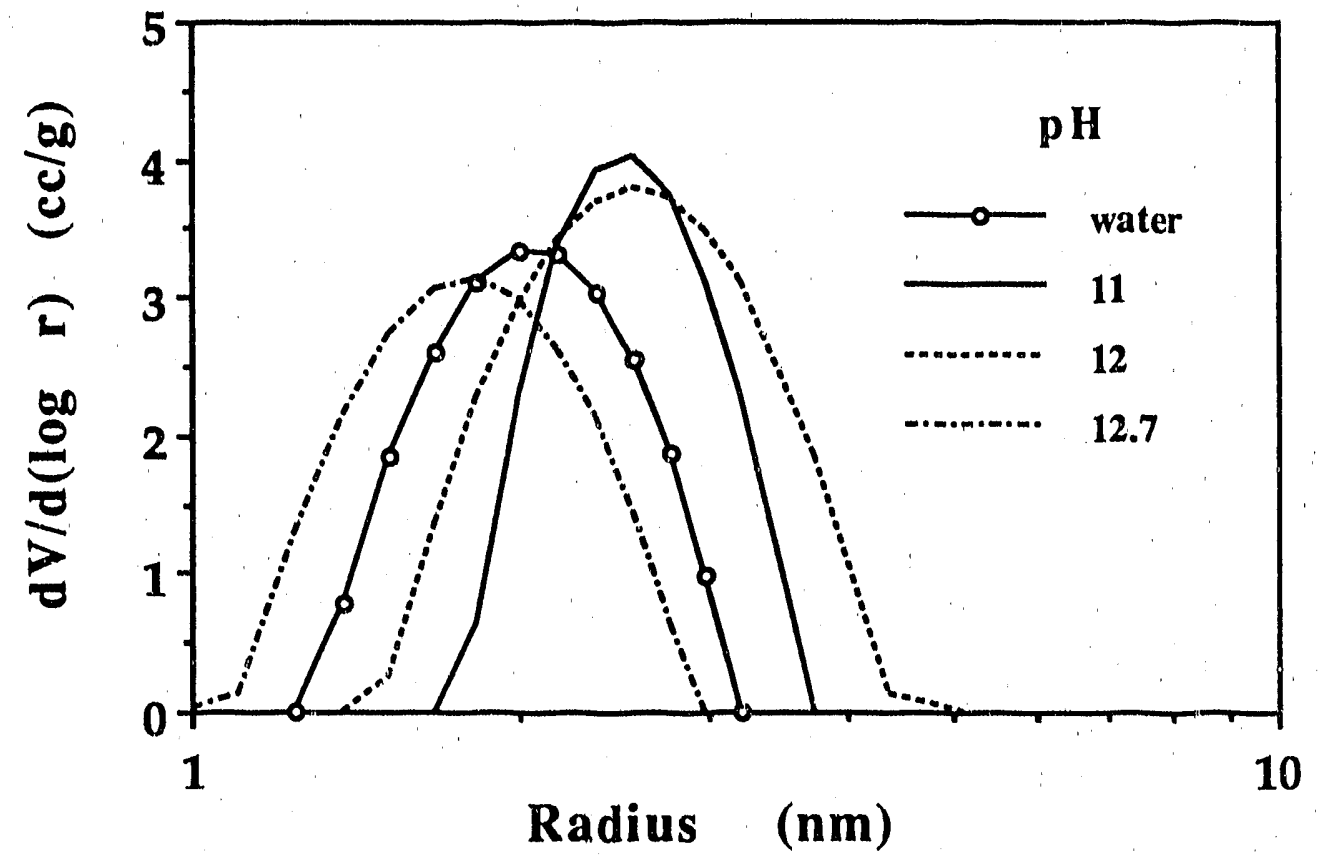

Figure 9 Effect of aging $\mathrm{B} 2$ gels in high $\mathrm{pH}$ pore fluids on xerogel pore structure (as measured with NMR).

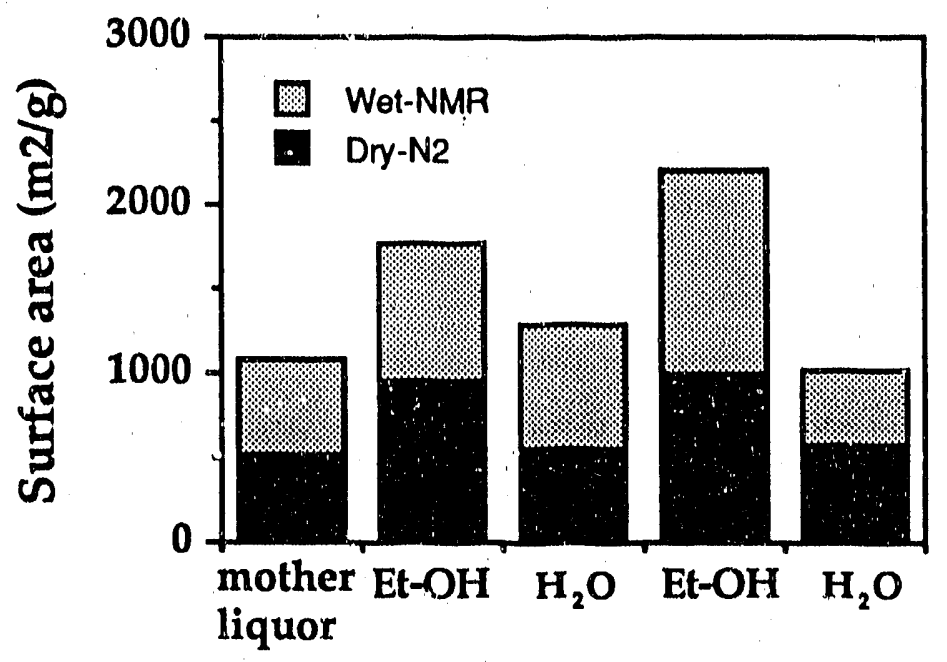

Figure 10 Variation of surface area (both wet and dry) for B2 gels as a function of the last fluid in which the gel was aged.

\section{ACKNOWLEDGEMENTS}

This work has been supported by Sandia National Laboratories (\#05-5795). PJD was partially supported by a New Mexico Fellowship for Underrepresented Groups in Science. The authors thank G. Johriston for N2 adsorption analysis, S. Thoma for sphere synthesis, and S. Hietala for SEM. 


\section{REFERENCES}

1. Schaefer, D.W., and Keefer, K.D., BETTER CERAMICS THROUGH CHEMISTRY I, Eds. Brinker, Clark, Ulrich, Materials Research Society Pittsburgh, ULTRASTRUCTURE PROCESSING OF ADVANCED CERAMICS, Eds. Mackenzie and Ulrich, J. Wiley and Sons, New York, (1988)

2. Keefer, K.D., in BETTER CERAMICS THROUGH CHEMISTRY I, Eds. Brinker, Clark, Ulrich, Materials Research Society, Pittsburgh, 15, (1984)

3. Brun, M., Lallemand, A., Quinson, J., and Eyraud, C., Thermochim. Acta, 1977, 21, 59.

4. Eyraud, C., Quinson, J.F., and Brun, M., in CHARACTERIZATION OF POROUS SOLIDS, Eds. Unger, Rouquerol, Sing, Elsevier, Amsterdam, (1988).

5. Gallegos, D.P., Munn, K., Smith, D.M., and Stermer, D.L., J. Colloid Interface Sci., 1987, 119, 127.

6. Bhattacharja, S., D'Orazio, F., Tarczon, J.C., Halperin, W.P., and Gerhardt, R., J.Am.Ceram.Soc., 1989, 72, 2126.

7. Brownstein, K.R., and Tarr, C.E., J. Mag. Resonance, 1977, 26, 17.

8. Ewing, B., Davis, P.J., Majors, P.D., Drobney, G., Smith, D.M., and Earl, W.E., J. Am. Ceram. Soc., in press.

9. Majors, P., Smith, D.M., and Davis, P.J., AlChE Journal, submitted.

10. Scherer, G.W., J. Non-Cryst. Solids, 1988, 100, 77.

11. Mizuno, T.. Nagata, H., and Manbe, S., J. Non-Cryst. Soliv's, 1988, $100,236$.

12. Brinker, C.J., and Scherer, G.W., SOL-GEL SCIENCE, Academic Press, New York, 1990.

13. Quinson, J.F., Dumas, J. Serughetti, J., J. Non-Cryst. Solids, 1986, $79,379$.

14. Quinson, J.F., Dumas, J., Chatelut, M., Serughetti, J., Guizard, C., Larbot, A., and Cot, L., J. Non-Cryst. Solids, 1989, 113, i4.

15. Glaves, C.L., Brinker, C.J., Smith, D.M., and Davis, P.J., Chem. Materials, 1989, 1, 34.

16. Brinker, C.J., Keefer, K.D., Schaefer, D.W., Ashley, C.S., J. NonCryst. Solids, 1982, 48, 47.

17. Stober,W., Fink, A., and E. Bohn, J.Colloid Interface Sci., 1968, 26, 62.

18. Gallegos, D.P., Smith, D.M., J. Colloid Interface Sci., 1988, 122, 143.

19. Davis, P.J., Gallegos, D.P., and Smith, D.M., Pow. Tech., 1987, 53, 39.

20. Axelous, M.A.V., Tchoubar, D., and Bottero, S.Y., Langmuir, 1989, 5, 1186.

21. Ner, R.K., THE CHEM!ISTRY OF SILICA, J. Wiley, New York, (1979). 


\title{
DISCLAIMER
}

\begin{abstract}
Whis report was prepared as an account of work sponsored by an agency of the United States Government. Neither the United States Government nor any agency thereof, nor any of their employees, makes any warranty, express or implied, or assumes any legal liability or responsibility for the accuracy, completeness, or useulness of any information, apparatus, product, or process disclosed, or represents that its use would not infringe privately owned rights. Reference herein to any specific commercial product, process, or service by trade name, trademark, manufacturer, or otherwise does not necessarily constitute or imply its endorsement, recommendation, or favoring by the United States Government or any agency thereof. The views and opinions of authors expressed herein do not necessarily state or reflect those of the United States Government or any agency thereof.
\end{abstract}



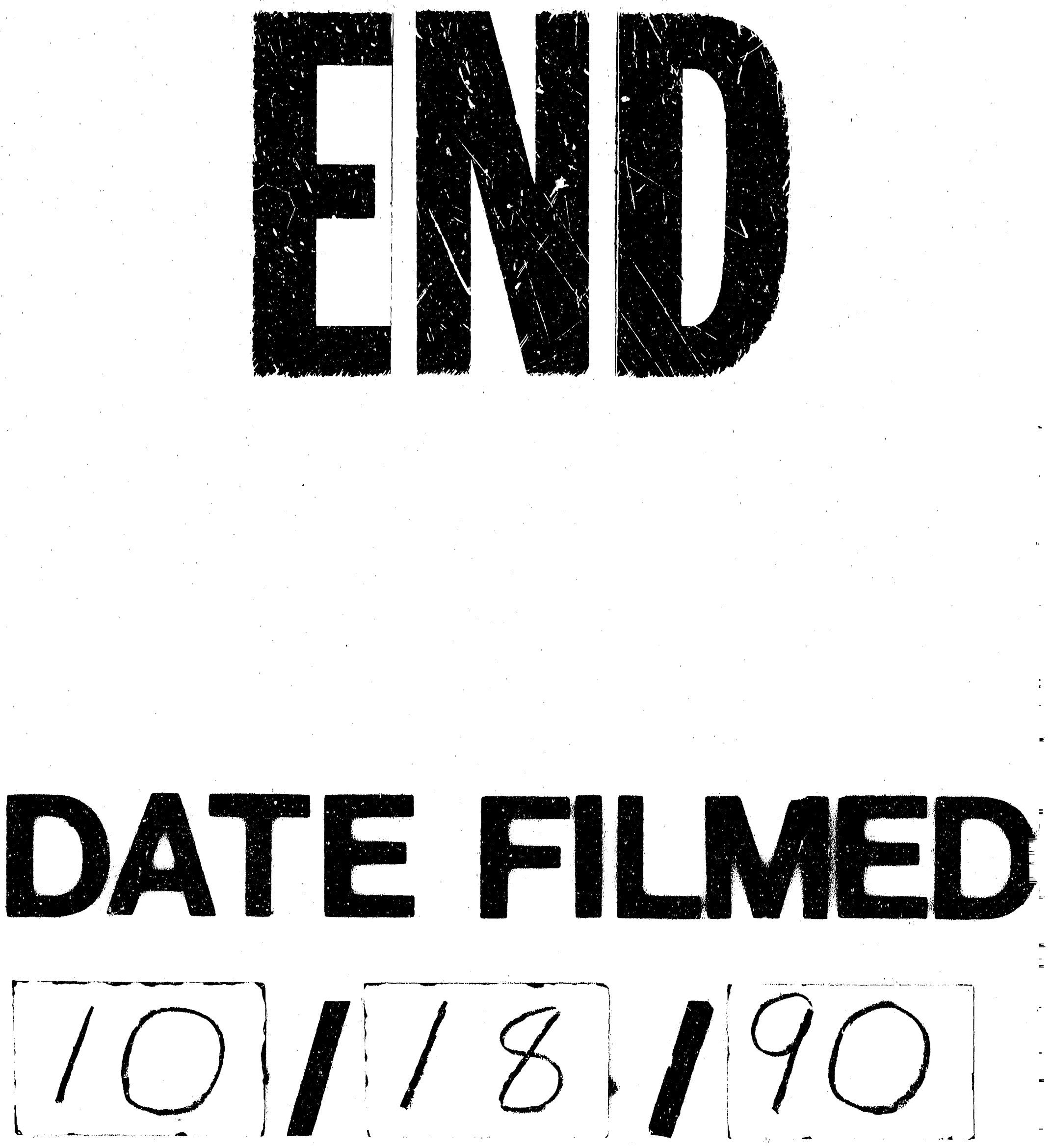
